\section{Kinetics of promoter Pol II on Hsp 70 reveal stable pausing and key insights into its regulation}

\author{
Martin S. Buckley, ${ }^{1,3}$ Hojoong Kwak, ${ }^{1,3}$ \\ Warren R. Zipfel, ${ }^{2}$ and John T. Lis ${ }^{1,4}$
}

${ }^{1}$ Department of Molecular Biology and Genetics, ${ }^{2}$ Department of Biomedical Engineering, Cornell University, Ithaca, New York 14853, USA

The kinetics with which promoter-proximal paused RNA polymerase II (Pol II) undergoes premature termination versus productive elongation is central to understanding underlying mechanisms of metazoan transcription regulation. To assess the fate of Pol II quantitatively, we tracked photoactivatable GFP-tagged Pol II at uninduced Hsp70 on polytene chromosomes and showed that Pol II is stably paused with a half-life of $5 \mathrm{~min}$. Biochemical analysis of short nascent RNA from $\mathrm{Hsp} 70$ reveals that this half-life is determined by two comparable rates of productive elongation and premature termination of paused Pol II. Importantly, heat shock dramatically increases elongating Pol II without decreasing termination, indicating that regulation acts at the step of paused Pol II entry to productive elongation.

Supplemental material is available for this article.

Received September 30, 2013; revised version accepted December 3, 2013.

Many metazoan genes have a high occupancy of transcriptionally engaged RNA polymerase II (Pol II) paused near their promoters (Bentley and Groudine 1986; Rougvie and Lis 1988; Muse et al. 2007; Zeitlinger et al. 2007; Core et al. 2008). Increasingly, studies indicate that the transition of this promoter-proximal Pol II into productive elongation is one of the major regulatory checkpoints of gene expression. Previous studies hypothesized that the accumulated Pol II at the promoter is either stably paused or iteratively terminating prematurely during early elongation (Bentley and Groudine 1986; Rougvie and Lis 1988). Interestingly, recent reports indicate that both pausing (DSIF and NELF) (Wu et al. 2003; Rahl et al. 2010) and termination (Dcp1a, Xrn2, and TTF2) (Brannan et al. 2012) factors are enriched at metazoan promoters, and their depletions can alter the distribution of Pol II. Therefore, the relative contribution of pausing or premature termination to promoter Pol II accumulation and its regulation is a central question that has yet to be quantitatively addressed in vivo.

[Keywords: RNA polymerase II; promoter-proximal pausing; termination; escape to productive elongation; photoactivation; nascent RNA]

${ }^{3}$ These authors contributed equally to this work.

${ }^{4}$ Corresponding author

E-mail jt110@cornell.edu

Article is online at http://www.genesdev.org/cgi/doi/10.1101/gad.231886.113.
The Drosophila Hsp70 heat-shock gene possesses a promoter-proximal Pol II that has been extensively characterized (Fuda et al. 2009). Under basal conditions, Pol II on Hsp70 pauses at sites $20-40$ base pairs (bp) downstream from the transcription start site (Rasmussen and Lis 1993; Kwak et al. 2013), producing an accumulation of Pol II at the $5^{\prime}$ end of the gene and a basal distribution of Pol II along the gene body (Lis 1998). Upon heat-shock induction, the escape of paused Pol II to productive elongation as well as the initiation rate increase up to 100 -fold, leading to the massive production of $H s p 70$ mRNA.

High-resolution live-cell imaging of the polytene chromosomes in Drosophila salivary glands provides one method to analyze the kinetics of Pol II induction and elongation during the heat-shock activation of Hsp 70 (Darzacq et al. 2009). The heat-shock-activated endogenous Hsp70 loci $(87 \mathrm{~A} / \mathrm{C})$ can be easily located in living polytene nuclei because they produce a distinct doublet of intense GFP-Pol II (or RFP)-containing puffs after the activation. Fluorescence recovery after photobleaching (FRAP) can then be used to measure dynamics and elongation rates (Yao et al. 2007). However, the dynamics of Pol II under uninduced conditions has been difficult to assay due to the challenges of locating the endogenous Hsp70 loci without heat-shock activation.

Notably, many features of the paused Pol II at the uninduced $H s p 70$ are similar to the large number $(\sim 70 \%)$ of active Drosophila genes containing paused Pol II (Core et al. 2012). In addition, many of the proteins identified to be involved in $H s p 70$ gene regulation have corresponding activities at other genes in various organisms (Fuda et al. 2009). These findings indicate that the mechanisms governing $H s p 70$ gene regulation are general. Therefore, in order to gain insights into the kinetic status of the promoter-proximal paused Pol II (paused Pol II that is stable vs. prematurely terminating), we measured the stability of paused Pol II at Drosophila Hsp70 through a combination of complementing optical and biochemical strategies that resolves previous challenges. We found that promoter-paused Pol II is relatively stable, and its entry to productive elongation, not its termination, is regulated by heat-shock activation.

\section{Results and Discussion}

To examine the kinetic fate of paused Pol II, we used an optical approach to measure the stability of Pol II fused to the photoactivatable GFP (paGFP) (Patterson and Lippincott-Schwartz 2002) at the Hsp70 locus on Drosophila salivary gland polytene chromosomes (Fig. 1A). These interphase-like giant chromosomes have been used as a platform for high-resolution imaging of the dynamics of Pol II and other transcription factors by optical pulsechase experiments at targeted genomic loci (Lis 2007). However, under uninduced basal conditions, identifying the endogenous Hsp70 gene loci is technically challenging, since the paused Pol II signal from Hsp70 is too weak to

(C) 2014 Buckley et al. This article is distributed exclusively by Cold Spring Harbor Laboratory Press for the first six months after the full-issue publication date (see http://genesdev.cshlp.org/site/misc/terms.xhtml). After six months, it is available under a Creative Commons License (Attribution-NonCommercial 3.0 Unported), as described at http:// creativecommons.org/licenses/by-nc/3.0/. 
A
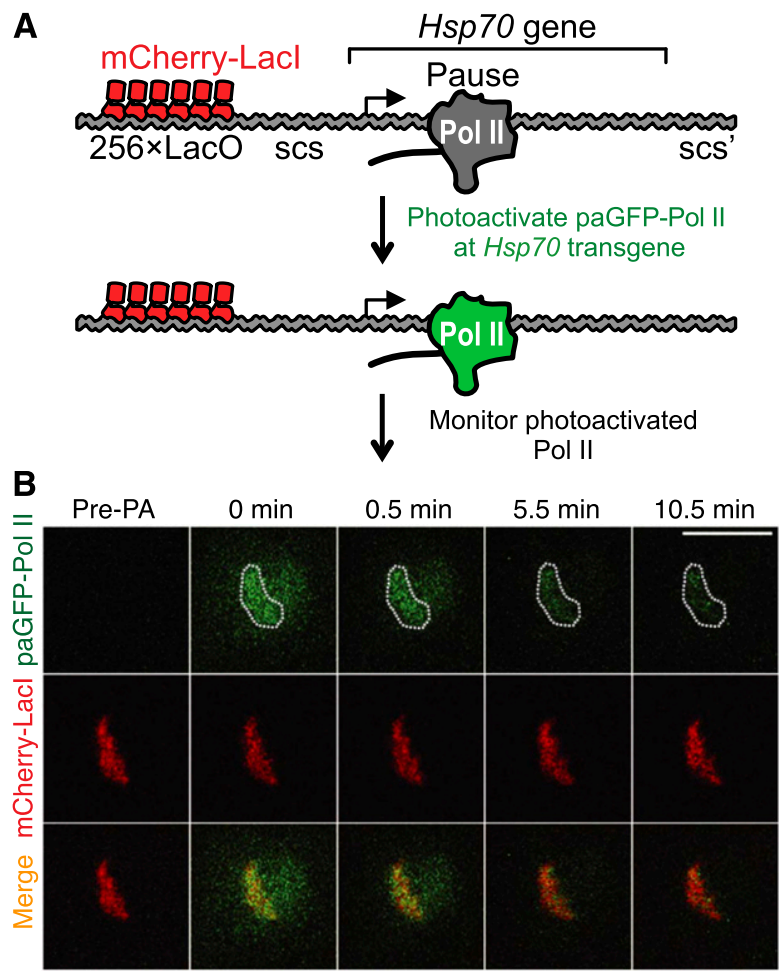

C

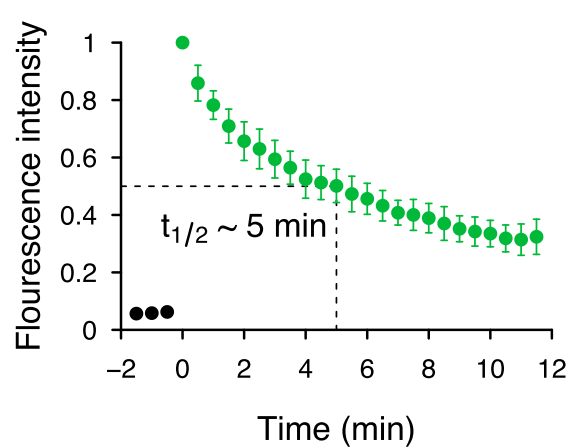

D

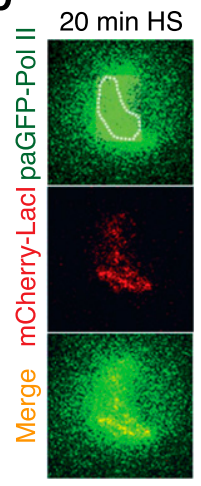

Figure 1. Imaging the stability of paused Pol II at the uninduced Hsp70 transgene in living cells. (A) Schematic of the live-cell imaging experiment showing mCherry-LacI bound to the operator sites (red) and photoactivated Pol II (green). Scs and scs' are the insulator elements flanking the $H s p 70$ gene. $(B)$ Uninduced mCherrymarked Hsp 70 transgene before and following the time course after paGFP-Pol II photoactivation. The region of interest is outlined by white dots. $(C)$ Normalized fluorescence intensities of paGFP-Pol II fluorescence decay after photoactivation (FDAP) under the uninduced condition $(n=9)$. (D) After heat shock, images of mCherryLacI, paGFP-Pol II (rephotoactivated after heat shock), and merge at the Hsp70 transgene. Error bars indicate SD. Bar, $10 \mu \mathrm{m}$.

allow it to be easily distinguished from other Pol II signals (Supplemental Fig. 1a).

To circumvent this problem, we generated a transgenic Hsp70 gene that can be easily identified on the polytene chromosomes for targeted analysis. The full-length Hsp70 gene is marked with 256 repeats of Escherichia coli Lac operator sites ( $\mathrm{LacO}$ ) and can be rapidly identified by coexpressing a fluorescently tagged Lac repressor (mCherry-LacI) in salivary gland nuclei (Fig. 1A). We tested that this LacO-tagged transgenic $H s p 70$ is functionally equivalent to the endogenous $\mathrm{Hsp} 70$ gene (Zobeck et al. 2010) by examining the recruitment and levels of Pol II intensity at puffs in response to heat shock (Supplemental Fig. $1 \mathrm{~b}, \mathrm{c})$. We also confirmed that the paGFP-labeled Pol II subunit (Rpb9) reliably tracks Pol II at both the endogenous $H s p 70$ loci and the LacO-tagged Hsp 70 transgene (Supplemental Figs. 2, 3).

The paGFP-Pol II is fluorescently inert at the Hsp70 transgene before photoactivation (Fig. 1B). To examine the dynamics of paused Pol II at the Hsp70 loci, we used laser-scanning confocal microscopy to specifically photoactivate paGFP-Pol II at the LacO-marked $\mathrm{Hsp} 70$ transgene under uninduced conditions (Fig. 1A). Figure 1, B and $\mathrm{C}$, shows the time series of the fluorescence decay of paGFP-Pol II at the $H s p 70$ transgene. Importantly, the fact that the paGFP-Pol II signal is heat-shock-inducible validates that the locus examined at the single mCherryLacI band was the $H s p 70$ transgene (Fig. 1D). Photobleaching is minimal during imaging (Supplemental Fig. 4), indicating that the decay of signal is due to the release of paGFP-Pol II from the transgene. The resulting decay approximates first-order kinetics with the half-life of $\sim 5$ min (Fig. 1C). Because the main form of Pol II at the uninduced Hsp70 locus is paused Pol II (Core et al. 2012), this clearance half-life of 5 min indicates that paused Pol II is relatively stable but has a finite lifetime (Table 1).

The lifetime of the paused Pol II can be a consequence of the escape into productive elongation, premature termination, or both. To measure the contribution of each to the stability of paused Pol II, we developed an independent biochemical kinetic strategy in Drosophila S2 cells (Fig. 2A). First, we evaluated the rate of escape into productive elongation. If a certain fraction of paused Pol II escape into productive elongation every minute $\left(k_{\mathrm{el}}\right)$, these escaped Pol II will be distributed in the gene body region defined by the speed of elongation. Therefore, the rate of escaping Pol II can then be derived from the speed of Pol II elongation and the relative ratio between gene body and paused Pol II density (Fig. 2B, ${ }_{i j}$ Supplemental Material). For this measurement, we used pre-existing nuclear run-on sequencing (GRO-seq or PRO-seq) data sets in S2 cells (Fig. 2C; Core et al. 2012; Kwak et al. 2013), and the elongation speed of $1.5 \mathrm{~kb} / \mathrm{min}$ for $\mathrm{Hsp} 70$ from previous studies (Yao et al. 2007; Ardehali and Lis 2009). The run-on sequencing results show that at Hsp 70 , an average of $86 \%$ of engaged Pol IIs are restricted to the promoter-proximal region, and $13 \%$ are distributed in the 2.4-kb gene body region (Fig. 2C). From these estimates, we calculated that $k_{\mathrm{el}}=0.094 \mathrm{~min}^{-1}$; that is, $33 \%$ of paused Pol II escape into elongation every $5 \mathrm{~min}$ (Table 1).

Having measured the amount of paused Pol II that escapes into productive elongation, we then devised a biochemical measurement of the Pol II that terminates prematurely. Paused Pol II that terminates will dissociate from chromatin, and the engaged short nascent RNA will be released (Fig. 2A). Therefore, the amount of free short nascent RNA relative to the chromatin-associated (paused) short nascent RNA will reflect the rate of premature termination (Fig. 2B). We measured the amount of short nascent Hsp70 RNA from chromatin-associated (paused) and free (terminated) Pol II using biochemical fractionation (Wuarin and Schibler 1994). Short nascent RNA was quantitatively measured by ligation-mediated quantitative RT-PCR (qRT-PCR) targeting most of the 
Buckley et al.

Table 1. Rate constants for the kinetics of promoter-proximal Pol II at uninduced Hsp70

\begin{tabular}{lllcrc}
\hline Method & Rate & \multicolumn{1}{c}{ Description } & Value $\left(\mathrm{min}^{-1}\right)$ & Half-life $(\mathrm{min})$ & Fate after 5 min \\
\hline Optical pulse chase & $k_{\mathrm{ctrl}}$ & Overall stability & $0.135(0.123-0.147)$ & $5.4(4.7-5.6)$ & $51 \%$ paused \\
& $k_{\mathrm{t}(\mathrm{FP})}$ & Elongation blocked by FP & $0.072(0.058-0.086)$ & $70 \%$ paused \\
Biochemical steady state & $k_{\mathrm{cl}}$ & Overall stability $\left(=k_{\mathrm{el}}+k_{\mathrm{t}}\right)$ & $0.149(0.095-0.165)$ & $4.7(4.2-7.3)$ & $47 \%$ paused \\
& $k_{\mathrm{el}}$ & Elongation by GRO-seq & $0.094(0.045-0.103)^{\mathrm{a}}$ & $33 \%$ elongated \\
& $k_{\mathrm{t}}$ & Termination in steady state & $0.055(0.035-0.075)$ & $19 \%$ terminated \\
\hline
\end{tabular}

The range estimate of the elongation rate was provided by Yao et al. (2007).

Hsp70 pausing region within 25-40 bp downstream from the transcription start site (Supplemental Fig. 5). The nascent RNA of the free fraction was, on average, $45 \%$ of the chromatin-bound fraction level in uninduced $H s p 70$ (Fig. 2D, UI), suggesting that a significant amount of Pol II may be terminating from the uninduced $H s p 70$ pause sites.

For a quantitative estimation of the paused Pol II termination rate, we analyzed the steady-state kinetics of short Hsp70 nascent RNA termination and decay (Fig. 2A; Supplemental Material). At steady state, the decay rate of the free nascent RNA should be equal to its production rate from the terminating Pol II. Therefore, the termination rate can be derived from the free RNA decay rate and the ratio between free and chromatinbound short nascent RNA (Fig. 2B). To estimate the decay rate, we blocked the RNA production at the initiation step by using Triptolide, a potent chemical inhibitor of the TFIIH helicase XPB (Titov et al. 2011), and measured the time course of free nascent RNA decay. The time course showed a decay half-life of $\sim 6 \mathrm{~min}$ and the decay constant $k_{\mathrm{d}}=0.123 \mathrm{~min}^{-1}$ (Fig. 2E). This decay constant, when combined with the ratio between free and chromatin-bound short nascent RNA (described above), allows the calculation of the termination constant $k_{\mathrm{t}}=0.055 \mathrm{~min}^{-1}$, meaning that $19 \%$ of paused Pol II terminate prematurely every $5 \mathrm{~min}$ (Table 1). The rate of clearing of paused Pol II is the sum of termination and escape to elongation; adding these rates gives a Pol II that has a clearance kinetic constant $\left(k_{\mathrm{cl}}\right)$ of $0.149 \mathrm{~min}^{-1}$, which corresponds to the half-life of $4.7 \mathrm{~min}$ (Table 1 ). Therefore, the two independent methods-biochemical steady-state kinetics and optical pulsechase measurements-are in close agreement with each other.

$\mathrm{P}-\mathrm{TEFb}$ (positive transcription elongation factor b) kinase activity is required for the escape of paused Pol II into productive elongation at most genes, including Drosophila Hsp70 (Lis et al. 2000; Chao and Price 2001; Ni et al. 2008; Rahl et al. 2010). To obtain an independent estimate of the early termination rate of paused Pol II in vivo, salivary glands were treated with the P-TEFb kinase inhibitor Flavopiridol /Chao and Price 2001; Ni et al. 2008) to block transcription elongation (Fig. 3A). Assaying the fluorescence decay after photoactivation (FDAP) of paGFP-Pol II at the uninduced $H s p 70$ transgene then reveals the loss of Pol II by mechanisms that are independent of $\mathrm{P}-\mathrm{TEFb}$ activity, and we assume this is mainly by termination (Fig. 3B). We further analyzed the fluorescence using a quantitative decay model in both control and Flavopiridol treatment (Supplemental Fig. 6). The decay curves show the stabilization of Pol II (Fig. 3C; Supplemental Fig. 7) and near doubling of the half-life with Flavopiridol treatment (Fig. 3D; Table 1), indicating that the elongation and termination can also be distinctly measured using optical methods in vivo. Together, the optical and biochemical analyses provide a strong indication that paused Pol II is relatively stable in uninduced cells but does undergo slow transitions to both productive elongation or premature termination in living cells. This is additionally supported by a very recent independent and different approach showing that many other active Drosophila genes also have stably paused Pol II (Henriques et al. 2013).

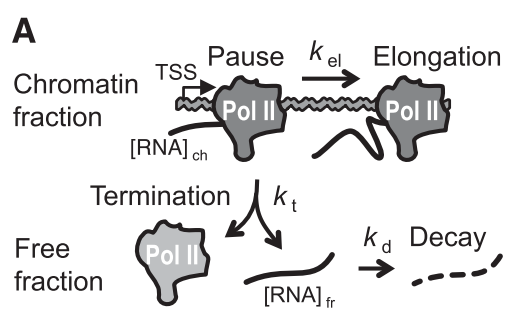

C

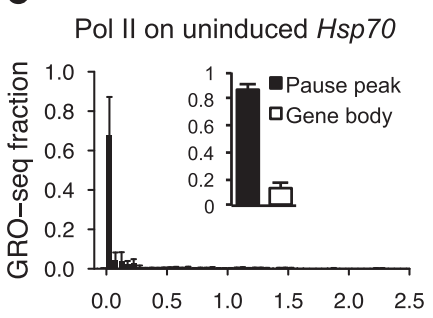

Position from Hsp70 TSS (kb)

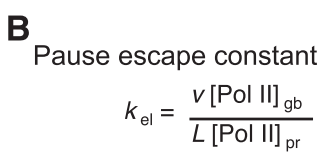

Termination constant

$$
k_{\mathrm{t}}=k_{\mathrm{d}} \frac{[\mathrm{RNA}]_{\mathrm{fr}}}{[\mathrm{RNA}]_{\mathrm{ch}}}
$$

E

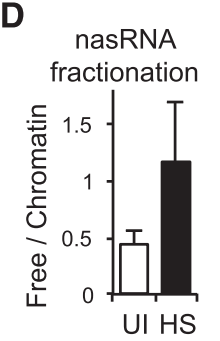

UI HS

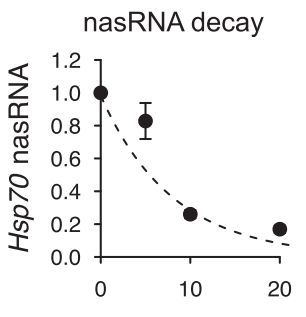

Decay time (min)
Figure 2. Biochemical analysis of steady-state paused Pol II kinetics. (A) Schematic showing the kinetic fates of paused Pol II and short nascent RNA (nasRNA). $\left(k_{\mathrm{el}}\right)$ Kinetic constant of paused Pol II elongation; $\left(k_{\mathrm{t}}\right)$ kinetic constant of paused Pol II termination; $\left(k_{\mathrm{d}}\right)$ short nascent RNA decay constant; $\left([\mathrm{RNA}]_{\mathrm{fr}}\right)$ free $H s p 70$ short nascent RNA; ([RNA $]_{\text {ch }}$ ) chromatin-bound $H s p 70$ short nascent RNA. (B) The equations used to calculate the pause Pol II elongation and termination rate constants (see the Supplemental Material). $(v)$ Elongation rate; $(L)$ gene length; ([Pol II $\left.]_{\mathrm{pr}}\right)$ Pol II fraction in pause region; $\left([\mathrm{Pol} \mathrm{II}]_{\mathrm{gb}}\right)$ Pol II fraction in gene body region. $(C)$ Estimation of $k_{\mathrm{el}}$ from GRO-seq (and PRO-seq) read fractions in $H s p 70$ (50-bp bins; $n=14$ independent data sets). The inset shows read fractions in pause region ( -50 to +250 from the transcription start site [TSS]) and gene body $(+300$ to $2.4 \mathrm{~kb})$. (D) Measuring the ratio between free and chromatin-bound short nascent RNA by qRT-PCR in uninduced (UI; $n=9)$ and 15 -min heat-shock (HS; $n=8)$ conditions. (E) Estimation of $k_{\mathrm{d}}$ from free Hsp70 short nascent RNA decay after Triptolide $(10 \mu \mathrm{M})$ addition (qRT-PCR). Each time point is normalized to the pretreatment level. $(C-E)$ Error bars indicate the SEM. 
A

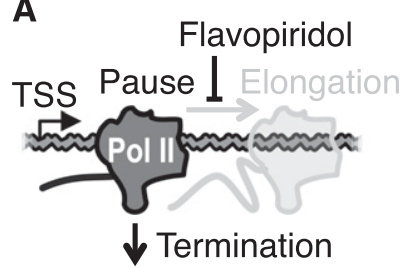

C

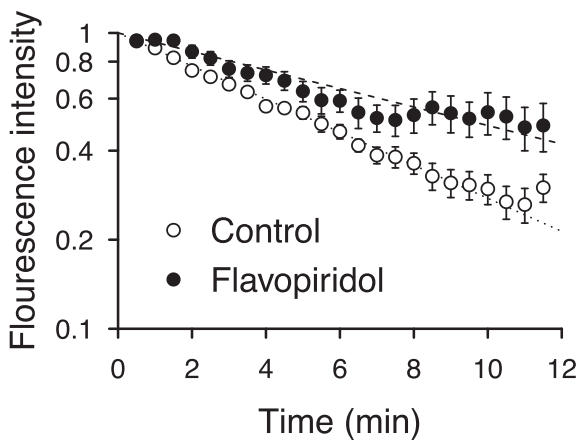

B

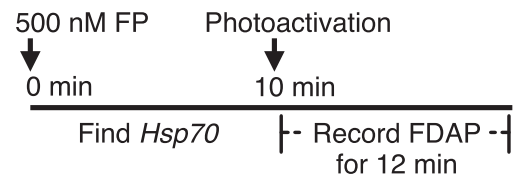

D control -Flavopiridol

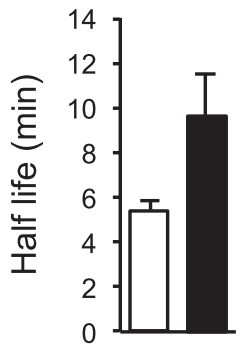

Figure 3. Optical measurement of the termination rate of paused Pol II at the Hsp70 transgene in living cells. (A) Schematic diagram outlining overall logic of using Flavopiridol to measure paused Pol II termination kinetics. (B) Illustration of the experimental scheme. $(C)$ Semi-log plot of normalized fluorescence intensities of paGFP-Pol II under uninduced condition in control $(n=9)$ and with Flavopiridol treatment $(n=7)$. Data are corrected for background Pol II signal (see the Materials and Methods). Error bars indicate SD. (D) Half-lives of paused Pol II decay with Flavopiridol treatment and control. $(C, D)$ Error bars indicate SEM.

The fact that paused Pol II can terminate raises the intriguing possibility that premature termination may play a role in $H s p 70$ gene regulation, as demonstrated in the examples of prokaryotic or viral promoters (Kao et al. 1987; Gollnick and Babitzke 2002). We assessed the contribution of premature termination to the regulation of $H s p 70$ expression level upon heat-shock induction based on our kinetic findings. The rate of Pol II escape into elongation rapidly increases after the heat-shock induction. This increase is equal to the initial rate of Pol II recruitment to the activated $H s p 70$, which was measured previously using a high-temporal-resolution recruitment study of Pol II in living cells (Zobeck et al. 2010), and here we estimate this rate to be about six molecules per minute per promoter (Fig. 4A). If all prematurely terminating Pol II are converted to productively elongating Pol II, the expected increase would be 0.055 molecules per minute per promoter (Table 1), which explains only a small fraction of the elongating Pol II (Fig. 4B). In addition, we observed an increase rather than the decrease of terminated nascent RNA fractions from paused Pol II upon heat shock (Fig. 2D, HS), opposite to the expectation if down-regulation of premature termination contributes to the heatshock induction. Collectively, these findings provide a comprehensive assessment of paused Pol II kinetics of elongation and termination on $H s p 70$ (Fig. 4C). Although cycles of initiation and premature termination occur at the promoter-proximal Pol II on Hsp70, this process is slow, and Pol II at the pause is relatively stable; the changes in
A
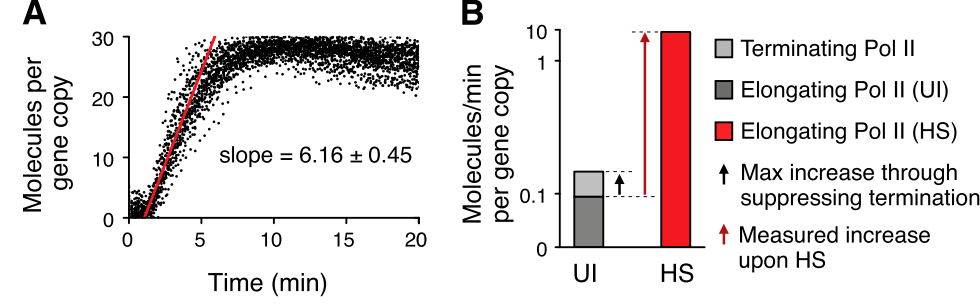

C
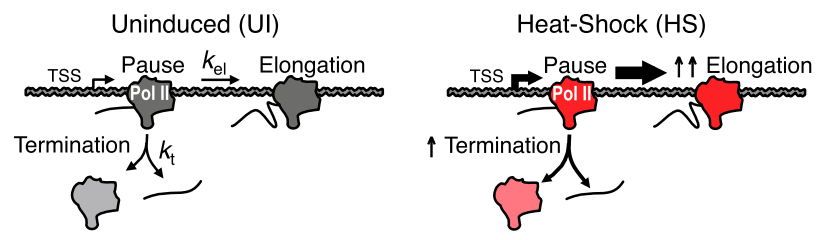

Figure 4. Kinetics of early elongating Pol II at Hsp70 during heat-shock induction. (A) Estimation of initial Pol II escape rate using live-cell imaging at the endogenous Hsp70 upon heat shock. The imaging data from a previous study (Zobeck et al. 2010) were analyzed $(n=33)$. (B) Contribution of premature termination to Pol II escape rate upon heat-shock induction. (UI) Uninduced condition; (HS) heat-shock induction. The $Y$-axis is in a nonlinear scale. (C) Model of early elongating Pol II kinetics under uninduced and heat-shock-activated conditions. The width of an arrow reflects the magnitude of the rate. 
The prolonged pausing itself may also lead to spontaneous Pol II termination, similar to what was observed in Pol II pausing at DNA damage sites (Somesh et al. 2005; Anindya et al. 2007). In addition, it is possible that a trailing or a newly initiating Pol II molecule may collide with the paused Pol II and result in the termination of the leading Pol II molecule, as seen in in vitro studies (Saeki and Svejstrup 2009). This may explain our observation of increased short terminated transcripts under heat-shock induction, where initiation is much more frequent. Thus, premature termination appears to be a consequence of the promoter dynamics rather than a control mechanism for Pol II productive elongation.

\section{Materials and methods}

\section{FDAP of polytene nuclei}

Intact Drosophila salivary glands were dissected from third instar larvae and transferred to Grace's medium. For drug experiments, glands were transferred to $500 \mathrm{nM}$ Flavopiridol (Sigma-Aldrich) diluted in medium. Laser-scanning confocal microscopy of salivary glands was carried out on a Zeiss 710 microscope. The mCherry-LacI-tagged $H s p 70$ transgene was identified using a 561-nm laser. Samples were photoactivated using a circular region of interest limited to the dimensions of the mCherryLacI spot using a 405-nm laser. The fluorescence of both the mCherry-LacI and Rpb9-paGFP was imaged using 561- and 488-nm lasers every $30 \mathrm{sec}$ for $12 \mathrm{~min}$. Photoactivation curves were normalized for the first image to equal 1. Half-lives were obtained by fitting the FDAP data to an exponential component using a grid-search regression in a mixed linear decay model. To confirm that the $H s p 70$ gene was targeted, an objective preheated to $37^{\circ} \mathrm{C}$ (Bioptechs) was used to heat-shock samples for $20 \mathrm{~min}$, and the locus was rephotoactivated.

\section{Biochemical steady-state kinetic analysis}

The rate constant of elongating Pol II from pausing $\left(k_{\mathrm{el}}\right)$ was derived from GRO-seq and PRO-seq data (Core et al. 2012; Kwak et al. 2013) in Drosophila S2 cells at the $H s p 70$ gene: $k_{\mathrm{el}}=v \lambda /[\mathrm{Pol} \mathrm{II}]_{\mathrm{pr}}$, where $v$ is the Pol II elongation speed (in kilobases per minute), $\lambda$ is the gene body Pol II density (in reads per kilobase), and $[\mathrm{Pol} \mathrm{III}]_{\mathrm{pr}}$ is the level of promoter-proximal Pol II (in reads). The kinetic constant of Pol II termination $\left(k_{\mathrm{t}}\right)$ was determined from nascent RNA fractionation in S2 cells: $k_{\mathrm{t}}=k_{\mathrm{d}}\left([\mathrm{RNA}]_{\mathrm{fr}} /[\mathrm{RNA}]_{\mathrm{ch}}\right)$, where $[\mathrm{RNA}]_{\mathrm{ch}}$ is the nascent RNA in chromatin fraction, $[\mathrm{RNA}]_{\mathrm{fr}}$ is the nascent RNA in free fraction, and $k_{\mathrm{d}}$ is the free nascent RNA decay constant. Nascent RNA fractionations were carried out as described previously (Wuarin and Schibler 1994), and a short spike-in RNA sequence from an Arabidopsis gene (RCP1) was added for the normalization after ligation-mediated qRTPCR analysis.

\section{Acknowledgments}

We thank Jie Yao (Yale University) for providing the range estimates of the elongation rate on $H s p 70$. This work was supported by a grant from the National Institute of Health (NIH; GM25232 to J.T.L.) and fellowships from NIH (GM087003 to M.S.B.) and the Howard Hughes Medical Institute (to H.K.).

\section{References}

Anindya R, Aygün O, Svejstrup JQ. 2007. Damage-induced ubiquitylation of human RNA polymerase II by the ubiquitin ligase Nedd4, but not Cockayne syndrome proteins or BRCA1. Mol Cell 28: 386397.

Ardehali MB, Lis JT. 2009. Tracking rates of transcription and splicing in vivo. Nat Struct Mol Biol 16: 1123-1124.

Bentley DL, Groudine M. 1986. A block to elongation is largely responsible for decreased transcription of c-myc in differentiated HL60 cells. Nature 321: 702-706.
Brannan K, Kim H, Erickson B, Glover-Cutter K, Kim S, Fong N, Kiemele L, Hansen K, Davis R, Lykke-Andersen J, et al. 2012. mRNA decapping factors and the exonuclease Xrn2 function in widespread premature termination of RNA polymerase II transcription. Mol Cell 46: 311-324.

Chao SH, Price DH. 2001. Flavopiridol inactivates P-TEFb and blocks most RNA polymerase II transcription in vivo. I Biol Chem 276: 31793-31799.

Core LJ, Waterfall JJ, Lis JT. 2008. Nascent RNA sequencing reveals widespread pausing and divergent initiation at human promoters. Science 322: 1845-1848.

Core LJ, Waterfall J, Gilchrist DA, Fargo DC, Kwak H, Adelman K, Lis JT. 2012. Defining the status of RNA polymerase at promoters. Cell Rep 2: $1025-1035$.

Darzacq X, Yao J, Larson DR, Causse SZ, Bosanac L, de Turris V, Ruda VM, Lionnet T, Zenklusen D, Guglielmi B, et al. 2009. Imaging transcription in living cells. Annu Rev Biophys 38: 173196.

Fuda NJ, Ardehali MB, Lis JT. 2009. Defining mechanisms that regulate RNA polymerase II transcription in vivo. Nature 461: 186-192.

Gollnick P, Babitzke P. 2002. Transcription attenuation. Biochim Biophys Acta 1577: 240-250.

Henriques T, Gilchrist DA, Nechaev S, Bern M, Muse GW, Burkholder A, Fargo DC, Adelman K. 2013. Stable pausing by RNA polymerase II provides an opportunity to target and integrate regulatory signals. Mol Cell 52: 517-528.

Kao SY, Calman AF, Luciw PA, Peterlin BM. 1987. Anti-termination of transcription within the long terminal repeat of HIV-1 by tat gene product. Nature 330: 489-493.

Kwak H, Fuda NJ, Core LJ, Lis JT. 2013. Precise maps of RNA polymerase reveal how promoters direct initiation and pausing. Science 339: 950953.

Laybourn PJ, Dahmus ME. 1990. Phosphorylation of RNA polymerase IIA occurs subsequent to interaction with the promoter and before the initiation of transcription. J Biol Chem 265: 13165-13173.

Lis JT. 1998. Promoter-associated pausing in promoter architecture and postinitiation transcriptional regulation. Cold Spring Harb Symp Quant Biol 63: 347-356.

Lis JT. 2007. Imaging Drosophila gene activation and polymerase pausing in vivo. Nature 450: $198-202$.

Lis JT, Mason P, Peng J, Price DH, Werner J. 2000. P-TEFb kinase recruitment and function at heat shock loci. Genes Dev 14: 792803.

Muse GW, Gilchrist DA, Nechaev S, Shah R, Parker JS, Grissom SF, Zeitlinger J, Adelman K. 2007. RNA polymerase is poised for activation across the genome. Nat Genet 39: 1507-1511.

Ni Z, Saunders A, Fuda NJ, Yao J, Suarez J-R, Webb WW, Lis JT. 2008. $\mathrm{P}-\mathrm{TEFb}$ is critical for the maturation of RNA polymerase II into productive elongation in vivo. Mol Cell Biol 28: 1161-1170.

Patterson GH, Lippincott-Schwartz J. 2002. A photoactivatable GFP for selective photolabeling of proteins and cells. Science 297: 18731877.

Rahl PB, Lin CY, Seila AC, Flynn RA, McCuine S, Burge CB, Sharp PA, Young RA. 2010. c-Myc regulates transcriptional pause release. Cell 141: $432-445$.

Rasmussen EB, Lis JT. 1993. In vivo transcriptional pausing and cap formation on three Drosophila heat shock genes. Proc Nat1 Acad Sci 90: 7923-7927.

Rougvie AEA, Lis JTJ. 1988. The RNA polymerase II molecule at the 5' end of the uninduced hsp70 gene of D. melanogaster is transcriptionally engaged. Cell 54: 795-804.

Saeki H, Svejstrup JQ. 2009. Stability, flexibility, and dynamic interactions of colliding RNA polymerase II elongation complexes. Mol Cell 35: 191-205.

Somesh BP, Reid J, Liu W-F, Søgaard TMM, Erdjument-Bromage H, Tempst P, Svejstrup JQ. 2005. Multiple mechanisms confining RNA polymerase II ubiquitylation to polymerases undergoing transcriptional arrest. Cell 121: 913-923.

Titov DV, Gilman B, He Q-L, Bhat S, Low W-K, Dang Y, Smeaton M, Demain AL, Miller PS, Kugel JF, et al. 2011. XPB, a subunit of TFIIH, is a target of the natural product triptolide. Nat Chem Biol 7: 182188 . 
Wu C-H, Yamaguchi Y, Benjamin LR, Horvat-Gordon M, Washinsky J, Enerly E, Larsson J, Lambertsson A, Handa H, Gilmour D. 2003. NELF and DSIF cause promoter proximal pausing on the hsp70 promoter in Drosophila. Genes Dev 17: 1402-1414.

Wuarin J, Schibler U. 1994. Physical isolation of nascent RNA chains transcribed by RNA polymerase II: Evidence for cotranscriptional splicing. Mol Cell Biol 14: 7219-7225.

Yao J, Ardehali MB, Fecko CJ, Webb WW, Lis JT. 2007. Intranuclear distribution and local dynamics of RNA polymerase II during transcription activation. Mol Cell 28: 978-990.

Zeitlinger J, Stark A, Kellis M, Hong J-W, Nechaev S, Adelman K, Levine M, Young RA. 2007. RNA polymerase stalling at developmental control genes in the Drosophila melanogaster embryo. Nat Genet 39: 15121516.

Zobeck KL, Buckley MS, Zipfel WR, Lis JT. 2010. Recruitment timing and dynamics of transcription factors at the Hsp70 loci in living cells. Mol Cell 40: 965-975. 


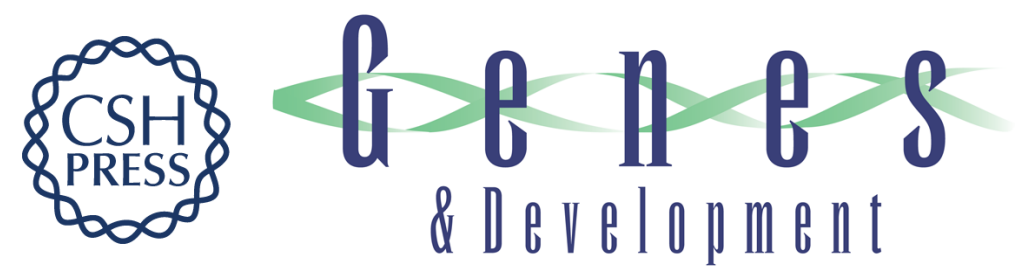

\section{Kinetics of promoter Pol II on Hsp70 reveal stable pausing and key insights into its regulation}

Martin S. Buckley, Hojoong Kwak, Warren R. Zipfel, et al.

Genes Dev. 2014, 28:

Access the most recent version at doi:10.1101/gad.231886.113

\section{Supplemental http://genesdev.cshlp.org/content/suppl/2014/01/06/28.1.14.DC1 Material}

References This article cites 31 articles, 11 of which can be accessed free at: http://genesdev.cshlp.org/content/28/1/14.full.html\#ref-list-1

Creative This article is distributed exclusively by Cold Spring Harbor Laboratory Press for the first Commons six months after the full-issue publication date (see

License http://genesdev.cshlp.org/site/misc/terms.xhtml). After six months, it is available under a Creative Commons License (Attribution-NonCommercial 3.0 Unported), as described at http://creativecommons.org/licenses/by-nc/3.0/.

Email Alerting Receive free email alerts when new articles cite this article - sign up in the box at the top Service right corner of the article or click here.

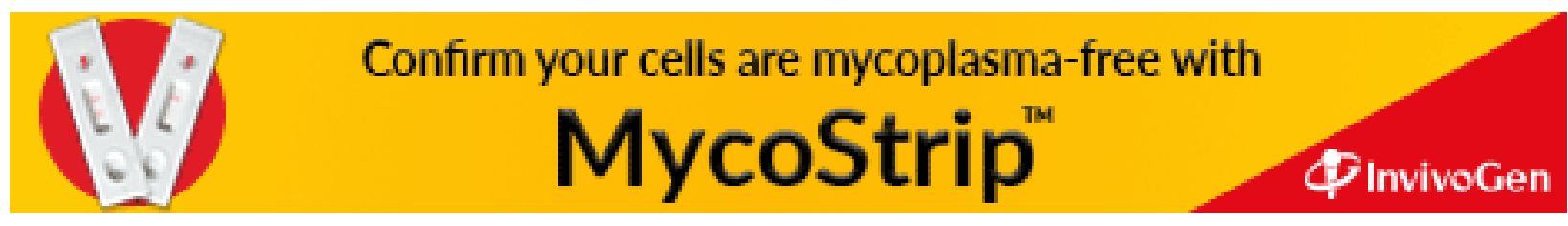

